\title{
RILKE, POETE, ADOLESCENT OU LA SOLITUDE ESSENTIELLE
}

\author{
Rilke, Poet, Adolescent or the Essential Solitude
}

FérodjaHocini ${ }^{l}$

\section{Résumé}

Rainer Maria Rilke, poète de la solitude par excellence, figure emblématique du poète sans patrie, fait revivre en nous des éprouvés adolescents, eux-mêmes reviviscences d'éprouvés primordiaux. Le thème de la solitude est cher au poète et traverse la plus grande partie de son œuvre, œuvre composée dans une alternance de moments d'exaltation euphorique et de profond désespoir. A la lumière d'une recherche biographique et des textes poétiques de Rilke, peuvent apparaître les origines et enjeux de cette solitude essentielle.

Motsclés: Rilke; Solitude; Création; Poésie; Narcissisme.

\section{Abstract}

Rainer Maria Rilke, pre-eminently poet of loneliness, emblematic figure of the home landless poet, makes us feel adolescent sensations, remembrances of primitive sensations. The theme of loneliness is very developed in Rilke's poetry and crosses more great part of his work, work made up in an alternation of moments of euphoric exaltation and of very deep despair. In the light of a biographical research and throughout the poetic texts of Rilke, one can see the origins and stakes of this essential loneliness appear.

Keywords: Rilke; Loneliness; Creation; Poetry; Narcissism.

"Car au fond et précisément pour l'essentiel, nous sommes indiciblement seuls."

(Rilke, Lettres à un jeune poète)

Psychanalyste - Paris, FR. e-mail: hocinif@yahoo.fr 
Rainer Maria Rilke est par excellence le poète de la solitude, figure emblématique du poète sans patrie, du poète adolescent. La découverte de Rilke et de sa poésie constitue souvent une rencontre. Et quelle rencontre! S 'éveillent alors en nous la sensation d'une re-trouvaille, d'une trace d'un témoignage, parfois aussi impression de déjà vu, déjà vécu, comme l'écho d'un sentiment d'intense solitude qui nous a profondément traversés, impression d'y lire peut-être alors quelque chose notre propre adolescence... Cette rencontre, Marguerite Yourcenar, dans une préface des Poèmes à la nuit, l'exprime à merveille:

Toute une partie de son œuvre m'échappe, s'enfonce pour moi dans le balbutiement et le brouillard, car les poèmes traduits ne sont jamais que les colombes auxquelles on a coupé les ailes, des sirènes arrachées à leur élément natal, des exilés sur la rive étrangère qui ne peuvent que gémir qu'ils étaient mieux ailleurs. A eux seuls, ses ouvrages en prose, ses lettres, quelques vers directement écrits en français, quelques écrits de gens qui l'ont aimé, ont suffi à $\mathrm{m}$ 'inspirer pour lui une tendresse infinie et fraternelle [...]. Il nous est cher parce que sa misère fut à peu près la nôtre, et que le sort lui a assigné la même portion de malheur. Les solutions qu'il a trouvées à sa vie partagée entre l'angoisse et le respect sont parmi celles que nous pourrions accepter, et cette communauté de péril et de solitude nous rend son génie un peu moins étranger (Yourcenar, 1994).

La solitude nous semble contingente au temps adolescent (à un certain temps en tout cas, au sens processuel du terme), une sorte d'espacetemps transitionnel nécessaire, pour reprendre l'expression winnicottienne, où éprouver (mettre à l'épreuve) la capacité d'être seul en présence de l'autre. (Winnicott, 1958/2001) Reviviscence ancienne, pour Mélanie Klein (Klein, 1959/1968/ , p. 121), " cet état de solitude intérieure résulte d'une aspiration universelle à connaître un état interne parfait, inatteignable. " Solitude des éprouvés aussi devant le réel du choc pubertaire, travail de séparation, de deuil des imagos parentales, de la remise en circulation des identifications. (Gutton, 1991).

Nous nous garderons bien ici de nous risquer à une étude de l'adolescence du poète, encore moins à une analyse de son œuvre et des mystères de la création « l'essence de la réalisation artistique nous est psychanalytiquement inaccessible $»$ disait Freud (1987, p. 167).

Il s'agira plutôt, dans le déroulement d'un fil associatif, de rechercher quel mouvement adolescent nous appelle dans cette poésie, d'entendre comment le jeune poète exprime cette détresse et cette solitude, formulant avec force des tentatives pour les dépasser.

\section{Solitude et poésie}

\author{
"Une chose est nécessaire : la solitude. \\ La grande solitude intérieure." \\ (Rilke, 1903, p. 61).
}

Rainer Maria Rilke confiait à Lou AndréasSalomé que depuis l'enfance, il connaissait d'expérience ce désert hostile qu'est la solitude. (Rilke, \& Andréas-Salomé, 1975) Ce thème est cher au poète et traverse une grande partie de son oeuvre. Eberhard Kretschmar, 1'un des principaux biographes de Rilke, distinguait un double aspect dans cette solitude: la Vereinsamung, l'esseulement pénible, et l'Einsamkeit, la retraite volontaire, envers et endroit du même écrin dont les replis enserrent la solitude à la fois angoissante et féconde du poète: « Aller en soi et, pendant des heures, ne rencontrer personne, -il faut pouvoir y arriver ». (Pitrou, 1938, p. 121) Cette solitude, le jeune poète la recherche en même temps qu'il la craint.

\section{Même si souvent solitaire - seul au monde, je ne le suis pourtant pas, car depuis longtemps déjà une [fidèle] amie m'a été envoyée.}

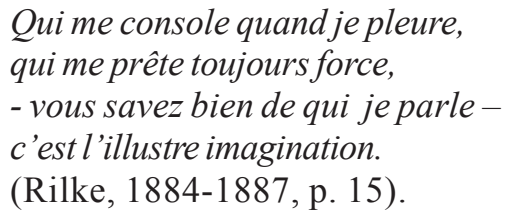

Rilke avait 19 ans quand il composa ce poème. C'est sa mère qui lui fit connaître la poésie, avant même qu'il sache lire. A sept ans, il commence à recopier des vers et apprend par cœur un grand nombre de longues ballades de Schiller. A 9 ans, il écrit un poème pour célébrer le mariage de ses parents (qui n'allaient pas tarder à se séparer). Sa 
santé est fragile, il est souvent malade. Il passe une petite enfance austère et malheureuse, au 19 de la Heinrichgasse, à Prague, auprès d'un père superficiel et velléitaire, Josef Rilke, modeste employé des chemins de fer (qui rêvait d'être officier et transmit la tristesse d'une ambition insatisfaite à son fils) et d'une mère pieuse bigote, Phia Enz. (Freedman, 1998). Elle habille son enfant en fille jusqu'à l'âge de sept ans, et même si l'usage est courant à cette époque dans les premières années de vie, on ne peut ignorer que sa première née était morte et que René Maria lui succédait dans une place d'emblée impossible. Dans ce poème de jeunesse intitulé La mère, il écrit :

\section{Tu m'as appelé mon enfant? \\ C'était un mot dans le vent. - Combien de hautes marches y a-t-il encore jusqu'à toi ? \\ Sa voix trouva les étoiles, mais ne trouva pas sa fille.}

\section{Dans la vallée au fond d'une taverne une dernière lampe s'est éteinte.}

\section{D’une solitude primordiale}

Alors pourquoi écrire? et « Ecrire quoi ?» s'interroge Jean-Yves Le Fourn (1994): « Si ce n'est ce point de jouissance quasi innommable », " Il y a en ce temps du sujet, impossible « ré-union » avec la jouissance d'antan, celle de l'Autre parental, celle-ci venant révéler que cette réponse sur le désir de la mère s'est effectuée dans un processus identificatoire qui comporte nécessairement une désexualisation [...] » (Le Fourn, 1994, p. 155). C'est le prix que vient payer le poète sur l'autel de la jouissance maternelle. Entre des périodes d'exaltation euphorique et de profond désespoir aux allures de cauchemars Brughéliens, il évoque, dans ses lettres à Lou Andréas-Salomé, sa souffrance, souffrance morale, souffrance du corps aussi, un corps blessé, torturé, transpercé, attaqué par des sensations mal unifiées:

Les alternances de tensions et de relâchement excessifs des tissus que j'éprouve aux tempes et dans la gorge s'étaient étendues à tout le corps, à croire qu'une envie de bâiller essayait de susciter dans chaque membre mille petites bouches pour s'y assouvir [...]. (Rilke \& Andréas-Salomé, 1975, p. 314).
Surpeuplement et désolation à la surface du corps comme dans l'univers hanté du poète: « Le sort a quelques fois de ces trous où l'on disparait; le mien s'appelle malaise, maladie [...] que sais-je » (Betz, 1937, p. 221-225 ). Lou, dans sa biographie, se souvient:

[...] au lieu de ce travail de préparation où l'artiste hésite avant l'action, toutes sortes de manifestations hyperesthésiques et d'irritation survenaient, des douleurs provoquant même des crises et s'étendant à tout le corps. Rainer appelait cela, parfois en plaisantant, mais la plupart du temps cela le tourmentait jusqu' au désespoir, sa 'productivité mal placée', et son corps un 'singe de l'esprit'. (Andréas-Salomé, 1977, p. 136).

Ailleurs, dans les Cahiers de Malte Laurids Brigge, fasciné, le poète s'identifie à un homme choréique au corps violenté par des décharges brutales dont il tente désespérément de maîtriser les mouvements anarchiques, en vain: « J'aurais pu encore continuer ma route. Mais à quoi bon? J'étais vide. Comme un papier vide, je traînais à la dérive $[\ldots] \gg$ Se décline à nouveau la métaphore du corps délié, mal unifié, dans une perte d'identité terrifiante où la peau ne peut garantir cette enveloppe contenante. On songe aux premiers temps de la vie, à la fonction du holding maternel, à la maintenance et à la contenance psychiques telle que les formule. (Anzieu, 1995, p. 121).

De cette mère justement, Rilke livrera à Lou cette violente confession:

Je ne la vois que rarement, mais, tu le sais, chacune de nos rencontres est une sorte de rechute. Chaque fois qu'il me faut revoir cette femme égarée, irréelle, sans lien avec rien et qui ne peut vieillir, j'ai le sentiment que tout enfant déjà, $j$ 'ai dû chercher à la fuir; et tout au fond de moi, après des années et des années d'allées et venues, je crains de n'être pas encore assez loin d'elle, de garder encore quelque part en moi des mouvements qui soient l'autre moitié de ses gestes étriqués, quelques fragments des souvenirs qu'elle emporte partout, en morceaux avec elle; alors je prends en horreur sa piété distraite, sa foi têtue, tout cela de difforme et de contrefait à quoi elle se cramponne, elle-même pareille à un vêtement vide, fantôme effrayant. (Rilke \& Andréassalomé, 1975, p. 131). 
Toute l'œuvre de Rilke ressemble à ce cri désespéré dans la tentative de restaurer cette enveloppe contenante, trouver un corps nouveau, une identité nouvelle, un nom nouveau.

\section{Le solitaire et son double}

Les Premiers poèmes (écrits entre l'âge de 9 et 22 ans) sont signés René Maria [Caesar] Rilke, Caesar dont la prononciation en allemand est très proche du mot Kayser, l'empereur. Son premier poème conservé, adressé à ses parents en 1884, est signé « Hannibal, général des Carthaginois », ${ }^{2}$ ailleurs c'est Napoléon qui est convoqué. A partir de l'été 1897, qui correspond à la date de sa rencontre avec Lou Andréas-Salomé, il change de prénom, oubliant celui de Caesar et métamorphosant René, il se fera dès lors appeler Rainer. Le jeune Rilke ne cesse d'être traversé par le désir irrépressible de se créer une origine supérieure et illustre, il rêve de couronnes poétiques et de reconnaissance:

\section{"Jele sais en rêve, et le rêve a raison: \\ j'aibesoind'espace comme toute une lignée" \\ ( Rilke, 1997a , p. 125).}

Dans cette pénible quête d'un double identificatoire, Rainer Rilke crée Malte Laurids Brigge, personnage à la fois héros et poète lui aussi en errance et profondément seul ${ }^{3}$ " Lorsqu' on parle des solitaires, on suppose toujours connues trop de choses. Non, ils ne le savent pas. Ils n'ont jamais vu un solitaire [...]». ( Rilke, 1911, p. 271) Quelle place occupent ces doubles? Peut-on en rapprocher les doubles créés à l'adolescence dans certaines expériences esthétiques, alors même qu'on ne peut mettre sur le même plan écriture de l'adolescent et productions de l'écrivain ou du poète, les mouvements pulsionnels à l'origine de la "création" étant différents dans leur but. Dans la perspective de l'expérience esthétique, on peut convenir, avec
Jean Guillaumin, qu'ils « servent dans leur principe, à égale distance du choix objectal et du choix narcissique, à assurer à l'adolescent des conteneurs et des appuis extérieurs, plus ou moins temporaires mais nécessaires ». (Guillaumin, 1997, p. 19). Or, c'est bien à ce carrefour que semble achopper l'univers rilkéen. Alain de Mijolla parle de l'écriture en termes «d'identification écran pour exprimer [les] vacillations narcissiques et [les] difficultés relationnelles »(Mijolla, 1986, p. 105-108), comparant l'écriture à un champ clos où se livrerait le combat des identifications contradictoires. (Oliveira, 1985). Pour Gutton:

L'écriture renvoie à un érotisme médiatisé avec l'autre, et à l'auto-érotisme. [...] Les deux modèles sont radicalement différents; intégrés aux processus d'adolescence, ils se complètent; la diversité des métapsychologies de l'écriture s'origine dans la proportion réciproque par laquelle ils s'intriquent. Sans doute pourraiton parler d'une fonction de l'écriture qui se modéliserait volontiers par l'identification projective dans ce qu'elle comprend de projection d'un contenu, de dialectisation de celui-ci avec un contenant extérieur (dont l'altérité d'objet est reconnu par le sujet), et de capacité identificatoire et identifiante. Elle serait le socle de la démarche d'écriture, à la fois ancrée sur des processus archaïques et permettant le travail de secondarisation. (Gutton, 1986, p. 63-64).

Cette frontière, nous l'avons évoquée dans la fragilité des limites des enveloppes corporelles, se manifestant aussi dans ce que la poésie de Rilke comporte de tourments et de replis comme le cœur d'une rose.

On perçoit là le reflet de la souffrance narcissique, la quête éperdue d'une unité primordiale et "entière" idéalisée dans la rose, rosecorps-de-mère-et-d'enfant selon l'expression rilkéenne qui symbolise à souhait ce rêve impossible et mortifère de la réunion première. Solitude essentielle de cette "vie" intérieure et figée en proie à une

\footnotetext{
pour la petite histoire, c'est le second point commun que Rilke partage avec Freud qui confiait à Fliessdans sa lettre de 3 décembre 1897 son "amour de collégien pour Hannibal", ce héros conquérant sémite; l'autre point commun étant bien sûr leur amitié avec Louise Andréas-Salomé.

3 sur le thème "'errance et solitude" voir le très beau texte de Olivia Alberti, 'Rilke, sans domicile fixe'.
} 
sédimentation chosifiante. Toute-puissance des mots, toute-puissance des idées, auto-suffisance d'un moi rempli de lui-même, soutenu, selon l'expression de François Richard, dans « son dédoublement et son redoublement par une écriture narcissique autoséductrice ». (Richard, 2001, p. 234).

Le narcisse fait place à la rose, la rose devient narcisse:

Tu étais assez riche, pour devenir cent

fois toi-même

en une seulefleur;

c'est l'état de celui qui aime...

Mais tu n'as pas pensé ailleurs.

Abandon entouré d'abandon,

tendresse touchant aux tendresses...

C'est ton intérieur qui sans cesse

se caresse, dirait-on;

se caresse en soi-même,

par son propre reflet éclairé.

Ainsi tu inventes le thème

du Narcisse exaucé.

(Rilke,1997b , p. 1126).

\section{Narcisse solitaire}

Dans un poème de la deuxième Elégie de Duino intitulé Narcisse, il écrit:

"Là, il n'est pas aimé. Là au fond,

Il n'y a que la froideur d'un lit de pierres bousculées,

Et je vois bien à quel point je suis triste.

Etait-ce là mon visage à ses yeux?

Cela nourrit-il donc son rêve

D'une douce crainte? Je sens presque la sienne. Car, à me perdre ainsi dans mon regard, Je pourrais me croire meurtrier."

(Rilke, 1997c, p. 559-560).

L'amour? Des femmes, nombreuses, ont croisé sa route, mais Rilke veut vivre le seul amour qui vaille dans l'absence et la solitude. Comme peut le suggérer le poème Narcisse, cette tentative éperdue de capturer l'objet en fait un objet haï, exigence meurtrière devant l'impossibilité de se fondre à cet objet, de devenir l'objet. Lou Andréas-Salomé, l'amante, la confidente et fidèle amie, l'a probablement compris (Peters, 1962/1968, p. 243-247):
Car, sans aucun doute, il y a dans tout processus artistique, quand on regarde au fond des choses, une part de danger, une part de rivalité avec la vie: pour Rainer, ce danger était encore plus prévisible parce que sa nature le poussait à maîtriser poétiquement ce qui était presque inexprimable, et à donner un jour la parole à l'indicible, grâce à la puissance de son lyrisme $[\ldots]$ je pris peur pour Rainer: il me semblait que n'importe quel genre de travail ou d'activités exercés sans aucune prétention vaudrait mieux que cette attente à vide pendant laquelle il se faisait de vains reproches (c'est ce qui l'angoissait le plus lui-même). [...] ce qui, sous forme de menace et de maladie, se faisait jour parfois comme étant le destin de Rainer, étaient accompagnés de moments magnifiques qui éveillaient les espoirs les plus inouïs. (Andréas-Salomé, 1977, p. 116).

L'un de ces moments "'inouïs", tournant décisif possiblement, survint à Duino où Rilke, composant les premières Elégies, fut projeté hors du cauchemar infernal du désespoir humain jusqu' aux plus hauts sommets de la création artistique.

\section{La Création contre l'enfer de la désolation?}

En 1911, Rilke vient d'achever les Cahiers de Malte Laurids Brigge. Cet achèvement le laisse dans la plus grande détresse. A Lou il écrit: "Peux-tu comprendre qu'après ce livre, je me suis fait l'effet d'un survivant, livré au désarroi le plus profond, désemparé, incapable d'œuvrer jamais plus? »( Rilke, 1911, p. 215). On pourrait à juste titre s'interroger sur la part non objectale de la perte qui accompagne l'achèvement de cette œuvre consacrée à son double, à une part de lui-même incontestablement. Tenter de représenter l'irreprésentable n'est pas sans danger. Maurice Blanchot, à sa manière, le rappelle en ces termes:

Parler poétiquement et disparaître appartiennent à la profondeur d'un même mouvement, que celui qui chante doit se mettre tout entier en jeu et, à la fin, périr, car il ne parle que l'approche anticipée de la mort; la séparation devancée, l'adieu donné par avance effacent en lui la fausse certitude de l'être, dissipent les sécurités protectrices, le livrent à une insécurité illimitée. (Blanchot, 2003/1955, p. 205). 
Le poète vacille sur la crête du précipice, dans cette exigence sans concession: faire que le mot soit au plus près de la chose, si près qu'il se confond à elle: le mot, non plus représentation de mot et représentation de chose, mais représentation-chose. Prendre le mot pour la chose est commun dans la psychose, la limite est mince, souligne François Gantheret (1989, p. 207-235) dans son analyse: « le poète frôle l'abîme. Ce qui l'en sauve, c'est de sans cesse en revenir, c'est de sans cesse garder le mouvement de ce voyage vers l'innommable. » Oui, Rilke décide d'en revenir, et c'est au prix d'un lourd constat, celui de l'incomplétude, (celui de la castration symbolique pourrait-on dire), d'une perte enfin consentie.

L'horizon s'ouvre alors sur les Elégies [...] Simple lecteur, on a le sentiment que quelque chose en effet s'est opéré (quelque chose qui n'est pas seulement du registre de la maturité aboutie du poète). "Ce qui maintenant se joue, poursuit François Gantheret, c'est le rapport de l'homme au monde et sa capacité de le créer, s'il accepte de se perdre, de s'y perdre, pour, peut-être, s'y retrouver autre ». (Gantheret, 1989, p. 226).

Loin de « la région perdue et désolée », lorsqu'il rencontre "sa vraie parole de poète" ${ }^{4}$ celle des Elégies, Rilke dira nettement que, dans cette nouvelle œuvre, à partir des mêmes données qui avaient rendu impossible l'existence de Malte, la vie redevient possible. Le poète apparaît enfin libéré du piège écrasant de la rose. La vie, ma vie, dira Rilke, est dehors...

\section{Référence}

Andréas-Salomé, L. (1977). Ma vie. Paris: PUF.

Anzieu, D. (1995). Le Moi-Peau. Paris: Dunod.

Blanchot, M. (2003-1955). Rilke et l'exigence de la mort. In M, Blanchot. L'espace littéraire. (pp. 151-210). Paris: Gallimard.

Betz, M. (1937). Rilke vivant: Souvenirs, lettres, entretiens. Paris: Emile-Paul Frères.

Freedman, R. (1998). Rilke, la vie d'un poète. Paris: Actes Sud.
Freud, S. (1987). Un souvenir de Léonard de Vinci. Paris: Gallimard.

Gantheret, F. (1989). Nulle part sans non: 1'Ouvert. Rilke: du narcissisme à la création du monde. Le temps de la réflexion. (pp. 207-235).

Guillaumin, J. (1997). Esthétique et identité. In T, Bayard. Adolescense. 15(1), 19. Paris: Greup

Gutton, P. (1986). De l'echec dans le succès de l'écriture. In: P, Gutton. Adolescense. (pp. 6364).Paris: Greup. 4, I.

Gutton, P. (1991). Le pubertaire. Paris: Le fil rouge.

Klein, M. (1959-1968). Se sentir seul. In M, Klein. Envie et gratitude et autres essais. (pp. 121) Paris: Gallimard.

Le Fourn, J. Y. (1994). Ecrire quoi? In J. Y, Le Fourn. Adolescense: Rencontre de l'ècriture. Actes du colloque AFAT. (pp. 155). Toulouse: Erès.

Mijolla, A. (1986). Fonction de l'ècriture dans l'adolescense. In A. Mijolla. Adolescense. (pp. 105-108). Greup. 4. I.

Oliveira, L. E. P. (1985). Etre seul avec un mort. Solitude et identification narcissique. In L. E. P. Oliveira. Dialogue. ( pp.03-15). AFCCC, 129.

Peters, H. F.(1962-1968). Ma sœur, mon épouse. (pp. 197-253). Paris: Gallimard.

Pitrou, R. (1938). Rainer Maria Rilke. Les thèmes principaux de son œuvre. Paris: Albin Michel.

Richard, F. (2001). L'écriture comme processus de subjectivation. In F. Richard. Le processus de subjetivation à l'adolescense. Paris: Dunod.

Rilke, R. M. (1884-1887). Poèmes épars. In M. R. Rilke. Oeuvres poétiques et théatrales. Paris: La plêiade.

Rilke, R. M. (1903). Lettres à un jeune poète. Paris. La plêiade.

Rilke, R. M. (1911). Les cahiers de Malte Laurids Brigge. Paris: La plêiade.

\footnotetext{
Selon l'expression de Blanchot
} 
Rilke, R. M. \& Andréas-Salomé, L. (1975). Correspondances. Paris: Gallimard.

Rilke, R. M. (1997a). Pour me fêter. In M. R. Rilke. Oeuvres poétiques et théâtrales. Paris: $\mathrm{La}$ Pléiade.

Rilke, R. M. (1997b). Les roses. In M. R. Rilke. Oeuvres poétiques et théâtrales. Paris: La Pléiade.

Rilke, R. M. (1997c). Narcisse. In M. R Rilke. Oeuvres poétiques et théâtrales. Paris: Gallimard.
Yourcenar, M. (1994). Préface aux poèmes à la nuit: Ranier Maria Rilke. Paris: Verdier.

Winnicott, D. W. (2001). La capacité d'être seul. In W. D. Winnicott. De la pédiatrie à la psychanalyse. (2a ed.). Paris: Payot. (1958).
Recebido em : 01/04/2007 Received in: 04/01/2007 Aprovado em : 20/05/2007 Approved in: 05/20/2007 